\title{
A Highly Efficient Aromatic Amine Ligand/Copper(I) Chloride Catalyst System for the Synthesis of Poly(2,6-dimethyl-1,4-phenylene ether)
}

\author{
Kisoo Kim ${ }^{1,2}$, Min Jae Shin ${ }^{1,3}$, Yong Tae Kim ${ }^{4}$, Joong-In Kim ${ }^{5}$ and Young Jun Kim ${ }^{1, *(D)}$ \\ 1 School of Chemical Engineering, Sungkyunkwan University, 2066 Seobu-ro, Jangan-gu, Suwon-si, \\ Gyeonggi-do 16419, Korea; kks0918@hyosung.com (K.K.); shinminjae@lgcare.com (M.J.S.) \\ 2 Hyosung R\&D Business Labs, 74 Simin-daero, Dongan-gu, Anyang-si 14080, Gyunggi-do, Korea \\ 3 LG Household \& Health Care R\&D Center, LG Science Park, Magok, Kangseo-go, Seoul 07795, Korea \\ 4 SAMSUNG SDI, SDI-dong, Maetan-dong 130, Samsung-ro, Yeongtong-gu, Suwon-si, \\ Gyeonggi-do 16678, Korea; yt74.kim@samsung.com \\ 5 Hyosung R\&D Business Labs, 74 Simin-daero, Dongan-gu, Anyang-si, Gyunggi-do 14080, Korea; \\ joonginkim@hyosung.com \\ * Correspondence: youngkim@skku.edu; Tel.: + 82-31-290-7317
}

Received: 19 February 2018; Accepted: 20 March 2018; Published: 22 March 2018

\begin{abstract}
Highly active catalyst systems for polymerizing 2,6-dimethylphenol were studied by using aromatic amine ligands and copper(I) chloride. The aromatic amine ligands employed were pyridine, 1-methylimidazole, 2-aminopyridine, 3-aminopyridine, and 4-aminopyridine. A mixture of chloroform and methanol $(9: 1, v / v)$ was used as a polymerization solvent. All experiments were performed with oxygen uptake measurement apparatus, while the reaction rate for each aromatic amine ligand-Cu catalyst system and the amount of by-product, $3,3^{\prime}, 5^{\prime} 5^{\prime}$-Tetramethyl-4, $4^{\prime}$ diphenoquinone (DPQ), were measured to determine the efficiency of the catalyst systems. The 4-aminopyridine/Cu (I) catalyst system was found to be extremely efficient in poly(2,6-dimethyl-1,4-phenylene ether) (PPE) synthesis; it had the fastest reaction rate of $6.98 \times 10^{-4} \mathrm{~mol} / \mathrm{L} \cdot \mathrm{s}$ and the lowest DPQ production. The relatively high basicity of 4-aminopyridne and the less steric hindrance arising from a coordination of $\mathrm{Cu}$ and 4-aminopyridine in this catalyst are responsible for the fast polymerization rate. When 2-aminoprydine (an isomer of 4-aminopyridine) was used as a ligand, however, no polymerization occurred probably due to steric hindrance.
\end{abstract}

Keywords: rate of polymerization; aromatic amine ligand; highly effective catalyst; copper(I) chloride; poly(2,6-dimethyl-1,4-phenylene ether); 3,3' $5,5^{\prime}$-tetramethyl-4,4'diphenoquinone

\section{Introduction}

Poly(2,6-dimethyl-1,4-phenylene ether) (PPE) is one of the most important engineering plastics, with a unique combination of mechanical properties, low moisture absorption, an excellent electrical insulation property, dimension stability, and inherent flame resistance [1]. Even though PPE has the melting temperature of $236{ }^{\circ} \mathrm{C}$, it is generally classified as an amorphous polymer with the glass transition temperature of $205{ }^{\circ} \mathrm{C}$ since its degree of crystallinity is as small as 3\% [2]. It is often processed with polystyrene due to its high softening point and high melt viscosity [3-5] and is widely used in many industrial applications, such as computer housings, television housings, keyboard frames, and interface boxes $[1,6,7]$.

Hay and co-workers have reported a synthesis method for PPE in which the monomer, 2,6-dimethyl phenol, in the $\mathrm{CuCl}$ /pyridine catalyst system yields high molecular weight PPE with 
the flow of $\mathrm{O}_{2}$ [8]. As shown schematically in Figure 1, the major product, PPE, is formed via C-O coupling and the minor product, $3,3^{\prime}, 5,5^{\prime}$-tetramethyl-4,4'diphenoquinone (DPQ), is formed via $\mathrm{C}-\mathrm{C}$ coupling at a yield less than $5 \%$ under proper polymerization conditions with the formation of the condensation product water [9].

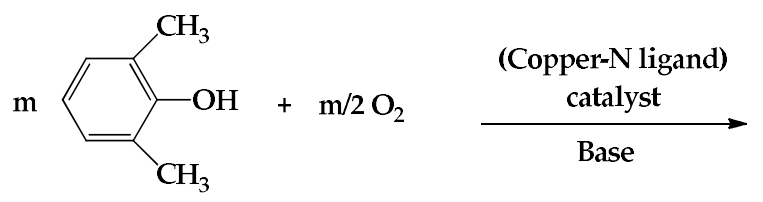

2,6-dimethyl phenol (2,6-DMP)

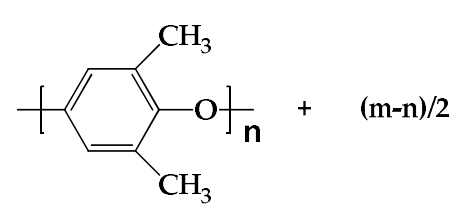

Polyphenylene ether (PPE) by $\mathrm{C}-\mathrm{O}$ coupling

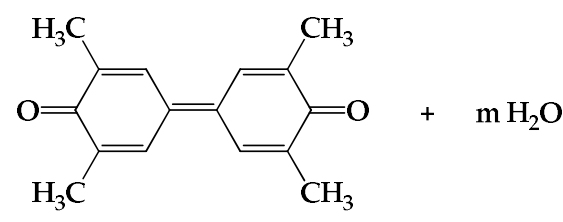

Diphenoquinones (DPQ) by C-C coupling

Figure 1. Synthesis of poly(2,6-dimethyl-1,4-phenylene ether) (PPE) by the copper-catalyzed oxidative coupling of dimethylphenol (2,6-DMP).

Although the synthesis mechanism has been studied by a significant number of researchers due to its excellent physical properties and being feasible, the mechanism actually has remained unclear. Proposed mechanisms are the radical mechanism, which involves free phenoxy radicals and coordinated phenoxy radicals, and the ionic mechanism, which involves ions such as phenoxonium cation $[10,11]$. Chloroform and toluene are generally used as polymerization solvents, and recently the usage of water as a solvent is being studied as one of the polymerization methods in respect of considering the environment [12-15]. Limiting the amount of DPQ production is critical, since the remaining DPQ in polymers plays a detrimental role that accelerates the decomposition of PPE while processing it at high temperature. For the ligands, aliphatic amines and imidazole and its derivatives are widely used due to their capability to bind with metal catalysts and the frequent occurrence of C-O coupling during PPE synthesis $[16,17]$. Studies have indicated that the remaining ligands in the polymers may lower the thermal stability of the PPE and change the color, especially when aliphatic amine ligands are used. This was verified by ${ }^{13} \mathrm{C}-\mathrm{NMR}$ spectroscopic analysis, and the most persuasive suggestion is that the quinone methide intermediate can be formed during PPE synthesis and it quickly reacts with an amine to remain in the products [18]. The incorporated amines could then deteriorate the thermal stability and cause a color change during processing.

Considering the previously mentioned aspects, different types of aromatic ligands were employed in this study instead of aliphatic amine, which can be contained in a polymer, to aim for developing a highly active aromatic amine/Cu catalyst system with a fast reaction rate and that is low in DPQ production.

\section{Experimental}

\subsection{Materials}

Highly purified (>99.9\%) 2,6-dimethylphenol (2,6-DMP) was provided by Aekyung Petrochemical (Daejeon, Korea) and copper(I) chloride ( $\mathrm{CuCl})(99.995 \%)$, chloroform $(99.8 \%)$, methanol $(99.8 \%)$, 
1-methylimidazole $(99 \%)\left(\mathrm{L}_{1}\right)$, 3-aminopyridine $(99 \%)\left(\mathrm{L}_{3}\right)$, pyridine $(99 \%)$, and citric acid $(99.5 \%)$ were purchased from Sigma Aldrich Korea (Seoul, Korea). 2-Aminopyridine (99\% pure) $\left(\mathrm{L}_{2}\right)$ was purchased from Alfa Aesar Korea (Seoul, Korea) and 4-aminopyridine ( $98 \%$ pure) $\left(\mathrm{L}_{4}\right)$ was purchased from Kanto Chemical Korea (Seoul, Korea). 3,3',5,5'-tetramethyl-4,4'diphenoquinone (DPQ) (98\%) was purchased from Sigma Aldrich and was recrystallized in a needle-shape with a mixed solvent of dichloromethane/n-hexane, 1:1 $(v / v)$, and dried overnight in a vacuum oven at $80^{\circ} \mathrm{C}$.

\subsection{Oxidative Coupling Polymerization of PPE and Oxygen Uptake Measurements}

All 2,6-DMP polymerization was implemented with the oxygen uptake measurement apparatus and an attached flask which was manufactured by our own laboratory [15]. Put 2,6-DMP $1 \mathrm{~g}$ ( 0.819 mmole), chloroform $9 \mathrm{~mL}$, and a magnetic stir bar into a $500 \mathrm{~mL}$ three-neck round-bottomed flask. Connect a dropping funnel to the three-neck round-bottomed flask, and put the reagents-containing flask into the $25{ }^{\circ} \mathrm{C}$ thermostat connecting to the oxygen uptake measurement equipment. Stir at $700 \mathrm{rpm}$ with oxygen flow at a rate of $330 \mathrm{cc} / \mathrm{min}$ in order to dissolve the monomer. After $5 \mathrm{~min}$, put $1 \mathrm{~mL}$ of the ligand-dissolved methanol into a dropping funnel and set the oxygen measurement equipment to a closed-system. As the ligands-dissolved methanol is transferring from the dropping funnel to the three-neck round bottomed flask, start the 2,6-DMP polymerization and let the reaction take place for two hours. Record the amount of oxygen uptake from the beginning to the end of the polymerization in 30-s intervals. After an hour of polymerization, the rate of oxygen uptake was very slow, but the polymerization was conducted for $2 \mathrm{~h}$ in a conservative manner. After $2 \mathrm{~h}$ of polymerization, precipitate the liquid products into the methanol with $1 \mathrm{~g}$ of citric acid dissolved in it, then retrieve. Dry overnight at $100{ }^{\circ} \mathrm{C}$ in a vacuum oven and collect the PPE powder. In order to investigate the effect of ligand and $\mathrm{CuCl}$ molar concentration on the polymerization reaction, the molar ratios of DMP, ligand, and $\mathrm{CuCl}$ have been varied as shown in Table 1.

Table 1. Mole ratios of 2,6-DMP, ligand, and $\mathrm{CuCl}$ employed in polymerization.

\begin{tabular}{ccccc}
\hline \multirow{2}{*}{ Condition } & \multirow{2}{*}{ 2,6-DMP (g) } & \multicolumn{3}{c}{ Molar ratios } \\
\cline { 3 - 5 } & & \multicolumn{3}{c}{ [2,6-DMP]:[Ligand]:[CuCl] } \\
\hline 1 & 1 & 200 & 70 & 2 \\
2 & 200 & 35 & 2 \\
3 & & 200 & 70 & 1 \\
\hline
\end{tabular}

\subsection{Measurement of $D P Q$ Content}

Since DPQ, the by-product of PPE synthesis, absorbs light in a wavelength of ca. $421 \mathrm{~nm}$, the amount of product was determined by measuring absorbance at $421 \mathrm{~nm}$ with ultraviolet (UV) spectroscopy $[19,20]$. The calibration curve was obtained from measuring UV absorbance at $\lambda_{\max }=421 \mathrm{~nm}$ of a DPQ at the concentration of $0.01,0.1,1,2$, and $4 \mathrm{ppm}$ in chloroform (Figure 2). After the reaction was completed, $0.1 \mathrm{~mL}$ of polymerization mixture in a liquid state was collected and weighed. Then, the polymerization mixture was diluted in $100 \mathrm{~mL}$ of chloroform for UV spectroscopic measurements and the absorbance was measured in order to analyze the DPQ contents in the samples based on the calibration curve. 


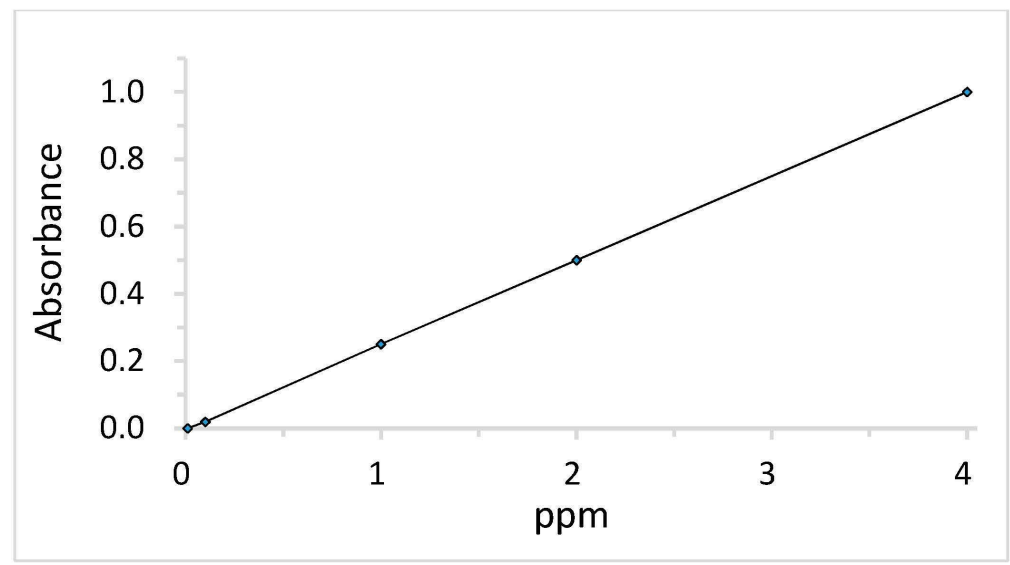

Figure 2. A plot of $3,3^{\prime}, 5,5^{\prime}$-Tetramethyl-4, $4^{\prime}$ diphenoquinone (DPQ) concentration (ppm) versus absorbance for determining wt \% of DPQ.

\subsection{Calculation of the Number of Moles of Oxygen Being Reacted during Polymerization Reaction}

Since the monomer, 2,6-DMP, and oxygen are reacting in a ratio of 2 to 1 , the number of moles of 2,6-DMP reacted can be calculated by measuring the oxygen uptake during the reaction, and then the reaction rate can also be determined by the moles of consumed 2,6-DMP. The density of oxygen gas at $25^{\circ} \mathrm{C}$ was calculated from the following Equation (1) [21], and the number of moles was obtained from the density.

$$
\mathrm{rO}_{2}^{t}=\left(\mathrm{rO}_{2}^{0} p\right) /\{760 \times(1+\alpha \mathrm{t})\}
$$

where $\mathrm{rO}_{2}^{t}=$ dioxygen density $(\mathrm{g} / \mathrm{L})$ at $\mathrm{t}^{\circ} \mathrm{C}, \mathrm{rO}_{2}^{0}=$ dioxygen density $(\mathrm{g} / \mathrm{L})$ at $0{ }^{\circ} \mathrm{C}(=1.429 \mathrm{~g} / \mathrm{L})$, $\alpha=$ thermal expansion coefficient $\left(=0.00367 \mathrm{~K}^{-1}\right)$, and $p=$ atmospheric pressure $(\mathrm{mmHg})$.

\subsection{Determination of Molecular Weight of the Polymers and Dilute Solution Viscosity}

The molecular weight of the polymers was measured by K-802, K-803, and K-804 (Shodex) columns attached to a gel permeation chromatography (GPC) with chloroform as a solvent at $30{ }^{\circ} \mathrm{C}$ in a flow rate of $0.5 \mathrm{~mL} / \mathrm{min}$. The molecular weight and polydispersity index (PDI), were then analyzed by using polystyrene standards (Waters). To determine the intrinsic viscosity ([ๆ]), the flow times of dilute solutions were measured using a Cannon-Ubbelohde viscometer (size 0 B) (State College, PA, USA) in $\mathrm{CHCl}_{3}$ at $30^{\circ} \mathrm{C}$.

\section{Results and Discussion}

\subsection{Effects of Chemical Structure of Ligand on the Rate of Polymerization}

Figure 3 shows plots of decrease in molar concentration of 2,6-DMP $(\mathrm{mmol} / \mathrm{L})$ versus reaction time (s) for $\mathrm{L}_{1}, \mathrm{~L}_{3}$ and $\mathrm{L}_{4}, \mathrm{~L}_{5}$, respectively, where $[2,6-\mathrm{DMP}]_{0}$ is the initial molar concentration $\left(=818.6 \mathrm{mmol} \mathrm{L}^{-1}\right)$ and [2,6-DMP] is the molar concentration given time. The rates of decrease in 2,6-DMP concentration were obtained from the slopes of the plots where linearity holds in a wide range. The rate of decrease in 2,6-DMP concentration also includes the rate of DPQ formation, which would be approximated to the rate of polymerization if the amount of DPQ produced is small. 

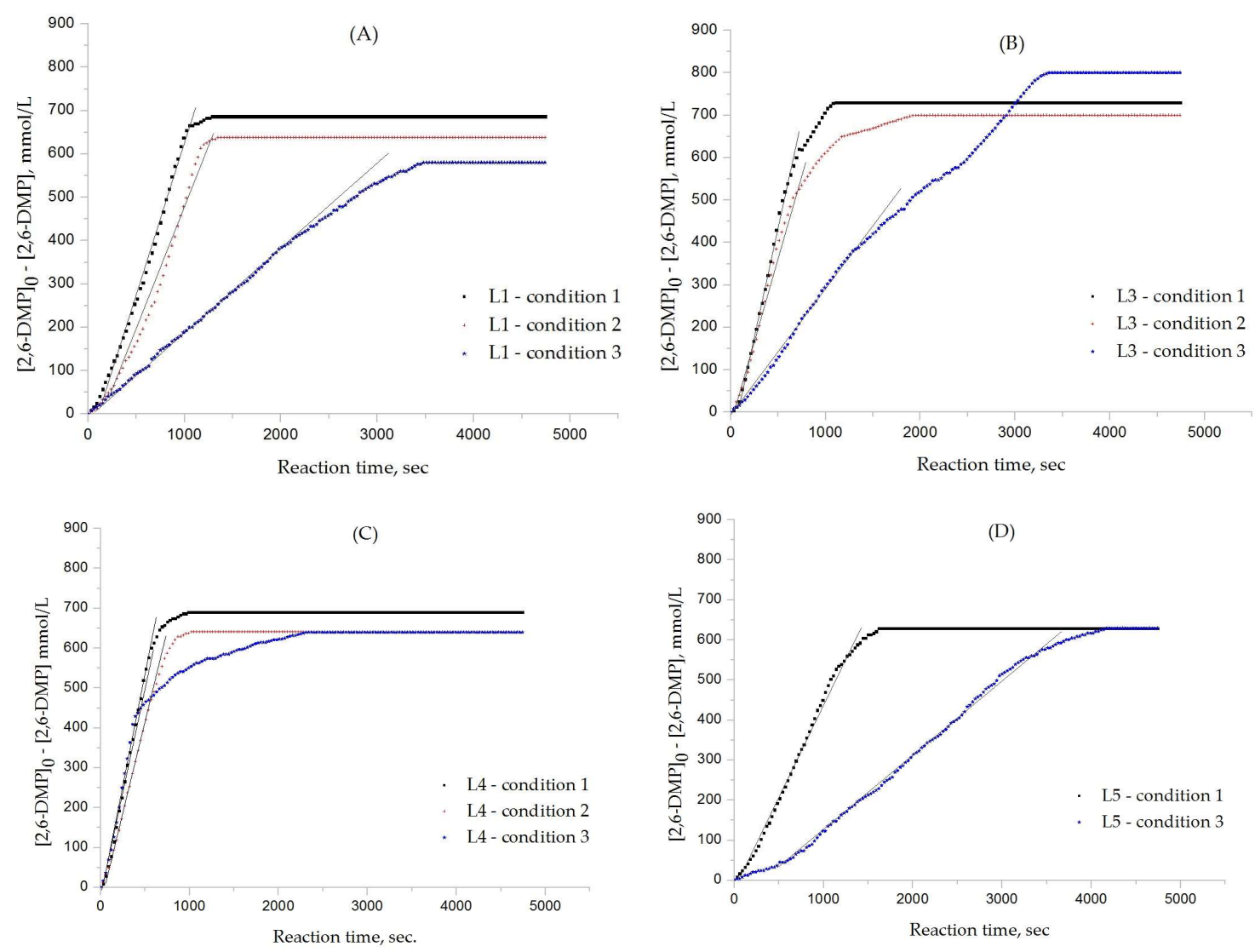

Figure 3. Plots of decrease in molar concentration of 2,6-DMP ( $\mathrm{mmol} / \mathrm{L})$ versus reaction time (s): (A) for $\mathrm{L}_{1} ;$ (B) for $\mathrm{L}_{3} ;$ (C) for $\mathrm{L}_{4} ;$ (D) for $\mathrm{L}_{5}$.

The effects of the chemical structure of each ligand on the rate of polymerization are presented in Table 2. These observations could be interpreted in view of $\mathrm{Cu}$ concentration, basicity, and steric effect of the ligands. For all the ligands used, the reaction rates tend to increase in the order of Condition 1,2, and 3. This means that the PPE polymerization rate is more affected by the amount of $\mathrm{Cu}$ catalyst under the experimental conditions performed than that of the ligands. As the ligand basicity increases, the polymerization rate tends to increase, except for $\mathrm{L}_{1}$ and $\mathrm{L}_{2}$, which have not polymerized. The ligands have not only affected the formation of the coordination complex with the $\mathrm{Cu}$ ion, but also the solvent basicity, which significantly changes the polymerization rate probably by affecting the oxidation potential of the phenol. A ligand with high basicity is strongly combined to the $\mathrm{Cu}$ metal pre-catalysts and it cannot be easily released. Therefore, amine ligands should have appropriate basicity in order to have high catalytic activity. The basicity of ligands is shown in Table 3. 
Table 2. Effects of chemical structure of ligands on the rate of polymerization.

\begin{tabular}{|c|c|c|c|c|c|}
\hline \multirow[b]{2}{*}{ Ligand } & \multicolumn{5}{|c|}{ Rate of Polymerization $\left(10^{-4} \mathrm{~mol} / \mathrm{L} \cdot \mathrm{s}\right)$} \\
\hline & $\mathrm{L}_{1}$ & $\mathbf{L}_{2}$ & $\mathrm{~L}_{3}$ & $\mathrm{~L}_{4}$ & $\mathrm{~L}_{5}$ \\
\hline \multicolumn{6}{|l|}{ Condition } \\
\hline 1 & 5.40 & $X^{*}$ & 6.53 & 6.98 & 3.89 \\
\hline 2 & 4.73 & $x$ & 3.57 & 6.58 & $x$ \\
\hline 3 & 1.68 & $x$ & 2.38 & 2.77 & 1.51 \\
\hline
\end{tabular}

$X *$ No polymerization.

Table 3. pKa values of amine ligands.

Ligands

The amino pyridine isomers $\mathrm{L}_{2}, \mathrm{~L}_{3}$, and $\mathrm{L}_{4}$ have conspicuously different effects on the polymerization rate. It is also observed that m-aminopyridine $\left(\mathrm{L}_{3}\right)$ or $\mathrm{p}$-aminopyridine $\left(\mathrm{L}_{4}\right)$ is a more effective ligand than $\mathrm{N}$-methylimidazole $\left(\mathrm{L}_{1}\right)$, which is perhaps the most widely employed aromatic ligand [15-17]. When $\mathrm{L}_{4}$ is used, the fastest polymerization rate of $6.98 \times 10^{-4} \mathrm{~mol} / \mathrm{L} \cdot \mathrm{s}$ is observed. Polymerization did not occur for $L_{2}$ although its basicity is similar to that of $L_{1}$ and a faster reaction rate was observed for $\mathrm{L}_{4}$ than for $\mathrm{L}_{3}$.

The effects of isomeric $\mathrm{L}_{2}, \mathrm{~L}_{3}$, and $\mathrm{L}_{4}$ ligands on the rate of polymerization are very different, which could be speculated on in view of steric effects [19]. Figure 4 shows the accessibility of a phenolate anion when $\mathrm{L}_{2}, \mathrm{~L}_{3}$, and $\mathrm{L}_{4}$, which are ortho-, meta-, and para-positioned, respectively, are coordinated to a $\mathrm{Cu}$ ion. The steric hindrance which occurs during the formation of the $\mathrm{Cu}$-amine ligand complex is a possible reason for the result. A possible active catalytic species in PPE synthesis is a coordination compound formed from two copper ions, amine ligands, and an aryloxide anion. When a $\mathrm{Cu}$-amine ligand complex is formed, the steric hindrance from the ligands would make it difficult for the phenolate anion to approach the $\mathrm{Cu}$ ion, and the reactivity would decrease as a result. In the case of $\mathrm{L}_{2}$, when the phenolate anion is about to coordinate with the Cu catalyst, it is subjected to steric hindrance from the amino group at the ortho position of $\mathrm{L}_{2}$. Therefore, it appears that in all of the experiments using $\mathrm{L}_{2}$ polymerization does not occur. Since $\mathrm{L}_{4}$ is the most basic ligand and free from steric hindrance in the formation of the coordination compound, it shows the highest reaction rate. $\mathrm{L}_{3}$ 
has a weaker basicity than $\mathrm{L}_{1}$, but leads to a larger polymerization rate. This observation could not be rationalized by steric hindrance since $\mathrm{L}_{3}$ would experience similar or less steric hindrance than $\mathrm{L}_{1}$ during a complex formation process. In fact, the rate of polymerization is greatly influenced by the type of solvent in addition to basicity and steric hindrance [25]. These two ligands differ in polarity: $\mathrm{L}_{1}$ is a polar aprotic compound and $\mathrm{L}_{3}$ is a polar protic compound. This difference seems to have affected the polymerization rate.

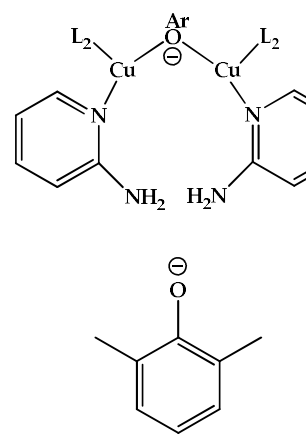<smiles>Cc1cccc(C)c1[O-]</smiles>

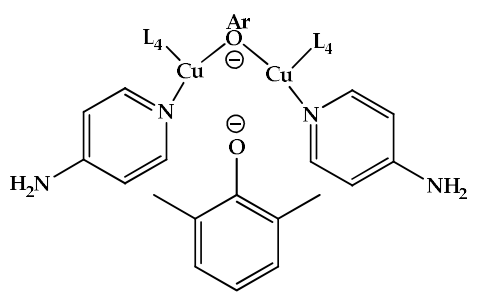

Where Ar is an aryl function group.

(a)

(b)

(c)

Figure 4. Steric environments of coordination compounds derived from $\mathrm{L}_{2}, \mathrm{~L}_{3}$, or $\mathrm{L}_{4}$ : (a) for $\mathrm{L}_{2}$, approach of the phenolate anion to the $\mathrm{Cu}$ ion is impossible due to strong steric hindrance (no polymerization); (b) for $\mathrm{L}_{3}$, approach of phenolate anion is possible due to reasonably relieved steric hindrance (polymerization occurs); (c) for $\mathrm{L}_{4}$, easy access of phenolate anion free from steric hindrance (fast polymerization occurs).

\subsection{Effects of Ligand and Polymerization Conditions on DPQ Formation and Molecular Weight of Polymer}

In Table 4, the amount of DPQ produced for each experiment is shown. The result of $L_{2}$ has been omitted for its not being polymerized. When $\mathrm{L}_{4}$ was used, the lowest DPQ formation was obtained at $2.2 \%$, and similar results were obtained under different experimental conditions. It is reported that the formation of DPQ is affected by various parameters, such as the polymerization temperature, types of solvent, polarity of reaction medium, and basicity of ligands, and its formation significantly increases as both solvent acidity and polymerization temperature increase $[8,16,26-28]$. DPQ production is the lowest with $\mathrm{L}_{4}$, which has the fastest reaction rate. This observation might be due to a low monomer concentration at the initial stage of polymerization as a result of the fast polymerization rate. As reported elsewhere [10], DPQ is largely produced at the beginning of the reaction when the concentration of the monomers is the highest since DPQ is formed by the C-C coupling of two 2,6-DMP monomers. It is also observed that the amount of ligand affects the production of DPQ more than the amount of Cu catalyst within the experimental conditions. The experimental result of Table 4 shows that the amount of DPQ formed is the biggest under Condition 2 for every ligand. It is presumed that the amine ligand changed the basicity of the solution and affected the production of DPQ.

Table 4. Weight \% of DPQ in PPE.

\begin{tabular}{ccccc}
\hline Condition & $\mathbf{L}_{\mathbf{1}}$ & $\mathbf{L}_{\mathbf{3}}$ & $\mathbf{L}_{\mathbf{4}}$ & $\mathbf{L}_{\mathbf{5}}$ \\
\hline 1 & 4.3 & 4.2 & 2.2 & 4.4 \\
2 & 10.4 & 7.7 & 2.8 & 15.7 \\
3 & 3.3 & 3.9 & 2.5 & 5.7 \\
\hline
\end{tabular}


The yield, viscosity, and molecular weight of produced PPE in each experiment are presented in Table 5. As previously mentioned, $\mathrm{L}_{2}$ and $\mathrm{L}_{5}$ have not polymerized in Condition 2, while PPE in high molecular weight could be obtained in all the other experiments, and the highest molecular weight was acquired with $\mathrm{L}_{3}$. In particular, $\mathrm{L}_{3}$, which has lower basicity than $\mathrm{L}_{4}$, resulted in high molecular weight polymers despite slow polymerization, which can be speculated on as follows. In fact, the 2,6-DMP polymerization is known to behave as a step-growth polymerization [29]. One of the important characteristics of the step-growth polymerization is that a high molecular weight polymer is achieved at the very end of polymerization. As the polymerization proceeds, the concentration of $\mathrm{OH}^{-}$ions formed from the equilibrium reaction of $\mathrm{H}_{2} \mathrm{O}$ and the ligand increases, and the greater the basicity of the ligand, the greater the concentration of $\mathrm{OH}^{-}$ions. At the end of the polymerization, the concentration of $\mathrm{OH}^{-}$can be significantly high, especially for the ligands having strong basicity since the concentration of fairly acidic phenolic $\mathrm{OH}$ groups ( $\mathrm{pKa} \sim 10.2$ ) is low. The $\mathrm{OH}^{-}$ions also play a role in lowering the oxidation potential of 2,6-DMP, which makes the oxidative polymerization easier. However, when the $\mathrm{OH}^{-}$ion concentration exceeds a certain level, the concentration of the active catalytic species decreases and the rate of polymerization decreases [30]. That is, in the case of $L_{4}$, which has higher basicity than $\mathrm{L}_{3}$, the polymerization rates were perhaps significantly lowered at the end of the polymerization by 'catalyst poisoning'. Thus, $\mathrm{L}_{4}$ resulted in lower molecular weight polymers than $\mathrm{L}_{3}$. $\mathrm{L}_{4}$ under Condition 2 (the condition with half the concentration) resulted in higher molecular weights than under Conditions 1 and $3 . \mathrm{L}_{5}$ resulted in higher molecular weights under Conditions 1 and 2 than $\mathrm{L}_{4}$. These observations can be explained for the same reason explained above. $\mathrm{L}_{3}$ resulted in higher molecular weights than the other ligands probably because it is an effective ligand leading to a fast reaction rate and the basicity was not too large, so catalyst poisoning did not occur until the end of the reaction.

Table 5. Effects of ligand and polymerization condition on molecular weight of PPE.

\begin{tabular}{ccccccc}
\hline Ligand & Condition & Yield (\%) & Intrinsic Viscosity $(\mathbf{d L} / \mathbf{g})$ & $\boldsymbol{M}_{\boldsymbol{n}}$ & $\boldsymbol{M}_{\mathbf{w}}$ & PDI \\
\hline \multirow{2}{*}{$\mathrm{L}_{1}$} & 1 & 74.6 & 0.63 & 12,400 & 36,800 & 2.97 \\
& 2 & 75.6 & 0.60 & 8700 & 22,800 & 2.62 \\
& 3 & 69.5 & 0.20 & 8100 & 13,500 & 1.67 \\
\hline \multirow{2}{*}{$\mathrm{L}_{2}$} & 1 & $\mathrm{X} *$ & $\mathrm{X}$ & $\mathrm{X}$ & $\mathrm{X}$ & $\mathrm{X}$ \\
& 2 & $\mathrm{X}$ & $\mathrm{X}$ & $\mathrm{X}$ & $\mathrm{X}$ & $\mathrm{X}$ \\
& 3 & $\mathrm{X}$ & 0.84 & 15,400 & 44,000 & 2.86 \\
\hline \multirow{2}{*}{$\mathrm{L}_{3}$} & 1 & 82.6 & 0.39 & 14,200 & 33,700 & 2.37 \\
& 2 & 74.6 & 1.02 & 16,100 & 44,500 & 2.76 \\
\hline \multirow{2}{*}{$\mathrm{L}_{4}$} & 3 & 96.7 & 0.45 & 9,060 & 31,500 & 3.48 \\
& 1 & 78.6 & 0.36 & 13,600 & 32,700 & 2.40 \\
& 2 & 72.6 & 0.29 & 12,700 & 29,400 & 2.31 \\
\hline \multirow{2}{*}{$\mathrm{L}_{5}$} & 3 & 72.6 & 0.40 & 12,400 & 29,400 & 2.37 \\
& 1 & 61.5 & $X$ & $X$ & $X$ & $X$ \\
& 2 & $X$ & 0.59 & 14,900 & 38,900 & 2.61 \\
\hline
\end{tabular}

$\mathrm{X}^{*}$ : No Polymerization. PDI: polydispersity index.

In Table 5, the PDI values appear in a wide range of 1.6-3.5. PDI values in a wide range have also been reported elsewhere [13]. It is not easy to explain this observation, since the polymerization mechanism is unclear at this point. However, it could be rationalized by considering the fact that the polymerization mechanism involves both a radical pathway and an ionic pathway [31], which may lead to molecular weight distributions different from those of conventional step-growth polymerization. 


\section{Conclusions}

In this study, PPE was synthesized under three conditions with various aromatic amine ligands, including 1-methylimidazole, which is widely used for PPE synthesis. As a result, the 4-aminopyridine/ $\mathrm{Cu}$ (I) catalyst system was the most efficient catalytic system for PPE synthesis as it had the fastest reaction rate and the lowest DPQ production under the experimental conditions. This is due to the adequate basicity of 4-aminopyridine and less steric hindrance as a phenolate anion approaches a $\mathrm{Cu}$ ion to form an active catalytic complex. With adequate basicity and less steric hindrance depending on the ligand structure, the reaction rate increases. Since the formation of DPQ usually occurs during an early stage of the reaction, it appears to have a lower amount of DPQ production as the reaction rate increases. The observed molecular weight dependence on polymerization conditions suggests that it is necessary not only to use a ligand leading to a fast reaction rate but also to control the basicity of the polymerization medium to prevent catalyst poisoning and to synthesize high molecular weight polymers.

Acknowledgments: This study was supported by the Technology Innovation Program (Industrial Strategic Technology Development Program, Contract No.: 10033616, 'Development of Techniques for PPE Molecular Structure Control and Production Process Thereof') funded by the Ministry of Trade, Industry \& Energy (MOTIE, Korea).

Author Contributions: Young Jun Kim and Yong Tae Kim conceived and designed the experiments; Kisoo Kim and Min Jae Shin performed the experiments and analyzed the data; Kisoo Kim wrote the paper; Joong-In Kim contributed reagents/materials/analysis tools.

Conflicts of Interest: The authors declare no conflict of interest.

\section{References}

1. Liang, M. Poly(phenylene oxide). In Handbook of Engineering and Specialty Thermoplastics; Thomas, S., Visakh, P.M., Eds.; Wiley: Hoboken, NJ, USA, 2011; Volume 3, pp. 15-53.

2. Karasz, F.E.; Bair, H.E.; O’Reilly, J.M. Thermodynamic properties of poly(2,6-dimethyl-1,4-phenylene ether). J. Polym. Sci. Part B 1968, 6, 1141-1148. [CrossRef]

3. Kim, D.K.; Song, K.H.; Koo, C.M.; Hong, S.M.; Chae, D.W. Characterization of compatibilized blends of nylon 66/poly(2,6-dimethyl-1,4-phenylene ether)/high-impact polystyrene filled with phosphinate-based flame retardants: Mechanical property, rheological behavior, and flame retardancy. J. Fire Sci. 2015, 33, 339-357. [CrossRef]

4. Jin, K.; Torkelson, J.M. Tg and Tg breadth of poly(2,6-dimethyl-1,4-phenylene oxide)/polystyrene miscible polymer blends characterized by differential scanning calorimetry, ellipsometry, and fluorescence spectroscopy. Polymer 2015, 65, 233-242. [CrossRef]

5. Lee, C.; Salehiyan, R.; Ham, D.S.; Cho, S.K.; Lee, S.J.; Kim, K.J.; Yoo, Y.; Hyun, K.; Lee, J.H.; Choi, W.J. Influence of carbon nanotubes localization and transfer on electrical conductivity in PA66/(PS/PPE)/CNTs nanocomposites. Polymer 2016, 84, 198-208. [CrossRef]

6. Li, Y.F.; Meng, J.; Zou, X.D. Flame Retardant Thermoplastic Composition Useful For Laser Direct Structuring. U.S. Patent 20130289178 A1, 23 May 2008.

7. Bussink, J.; van de Grampel, H.T. Poly(phenylene Oxide). In Industrial Polymers Handbook: Products, Processes, Applications, 2nd ed.; Wilks, E.S., Ed.; Wiley-VCH: Cham, Germany, 2001; Volume 3, pp. $1333-1348$. ISBN 978-3527302604.

8. Hay, A.S. Poly(2,6-diphenyl-1,4-phenylene oxide). Macromolecules 1969, 2, 107-108. [CrossRef]

9. Saito, K.; Pant, S.; Hearn, M.T.W. Oxidative polymerization of 2,6-dimethylphenol in water using bis-triazacyclononane copper catalyst. J. Appl. Polym. Sci. 2011, 122, 2174-2180. [CrossRef]

10. Aubel, P.G. Various Aspects of Copper-Catalysed Oxidative Coupling Reaction of 2,6-Dimethylphenol. Ph.D. Thesis, Universiteit Leiden, Leiden, The Netherlands, 19 June 2002.

11. Baesjou, P.J.; Driessen, W.L.; Challa, G.; Reedijk, J. Ab Initio calculations on 2,6-dimethylphenol and 4-(2,6-dimethylphenoxy)-2,6-dimethylphenol. Evidence of an important role for the phenoxonium cation in the copper-catalyzed oxidative phenol coupling reaction. J. Am. Chem. Soc. 1997, 119, 12590-12594. [CrossRef] 
12. Gu, C.; Zhu, J.; Shentu, B.; Liu, Q.; Weng, Z. Oxidative polymerization behavior of 2,6-dimethylphenol in aqueous media with potassium ferricyanide. Chin. J. Polym. Sci. 2009, 27, 543-549. [CrossRef]

13. Saito, K.; Kuwashiro, N.; Nishide, H. Catalyzed oxidative polymerization to form poly(2,6-dimethyl-1,4-phenylene oxide) in water using water-soluble copper complex. Polymer 2006, 47, 6581-6584. [CrossRef]

14. Liu, Q.; Wu, H.G. Carboxylic-supported copper complexes as catalyst for the green oxidative coupling of 2,6-dimethylphenol: Synthesis, characterization and structure. Comptes Rendus Chim. 2013, 16, 451-454. [CrossRef]

15. Kim, N.C.; Kim, Y.T.; Nam, S.W.; Jeon, B.S.; Kim, Y.J. Synthesis of poly(2,6-dimethyl-1,4-phenylene ether) with controlled molecular weight via suspension polymerization catalyzed by amine-copper (I) complexes under various reaction conditions. Polym. Bull. 2013, 70, 23-33. [CrossRef]

16. Sivek, R.; Bures, F.; Pytela, O.; Kulhanek, J. Imidazole-based Potential Bi- and Tridentate Nitrogen Ligands: Synthesis, Characterization and Application in Asymmetric Catalysis. Molecules 2008, 13, 2326-2339. [CrossRef] [PubMed]

17. Wei, C.; Gert, B.; Ger, C. Studies on immobilized polymer-bound imidazole-copper (II) complexes as catalysts. 3. Immobilization of copper (II) complexes of poly(styrene-co- $N$-vinylimidazole) by grafting on silica and their catalysis of oxidative coupling of 2,6-disubstituted phenols. Macromolecules 1991, 24, 3982-3987. [CrossRef]

18. White, D.M.; Nye, S.A. Carbon-13 NMR study of poly(2,6-dimethyl-1,4-phenylene oxide)s. Sites of amine incorporation. Macromolecules 1990, 23, 1318-1329. [CrossRef]

19. Li, K.T.; Shieh, D.C. Polymerization of 2,6-dimethylphenol with mixed-ligand copper complexes. Ind. Eng. Chem. Res. 1994, 33, 1107-1112. [CrossRef]

20. Chen, C.W.; Lin, I.H.; Lin, C.C.; Lin, J.L.; Horie, M. Synthesis of poly(2,6-dimethyl-1,4-phenylene oxide) derivatives in water using water-soluble copper complex catalyst with natural ligands. Polymer 2013, 54, 5684-5690. [CrossRef]

21. Baesjou, P.J.; Driessen, W.L.; Challa, G.; Reedijk, J. A kinetic and spectroscopic study on the copper catalyzed oxidative coupling polymerization of 2,6-dimethylphenol. X-ray structure of the catalyst precursor tetrakis (N-methylimidazole) bis(nitrato) copper(II). J. Mol. Catal. A Chem. 1996, 110, 195-210. [CrossRef]

22. Bruice, T.C.; Schmir, G.L. Imidazole Catalysis II The Reaction of substituted imidazoles with phenyl acetates in aqueous solution. J. Am. Chem. Soc. 1958, 80, 148-156. [CrossRef]

23. Angyal, S.J.; Angyal, C.L. The tautomerism of N-hetero-aromatic amines. Part I. J. Chem. Soc. 1952, 1461-1466. [CrossRef]

24. pKa Data Compiled by R. Williams. Available online: https://www.chem.wisc.edu/areas/reich/pkatable/ pKa_compilation-1-Williams.pdf (accessed on 10 August 2017).

25. Gamez, P.; Simons, C.; Steensma, R.; Driessen, W.L.; Challa, G.; Reedijk, J. A spectacular increase in the polymerization rate of 2,6-dimethylphenol induced by acetonitrile. Eur. Polym. J. 2001, 37, 1293-1296. [CrossRef]

26. Liao, B.S.; Liu, Y.H.; Peng, S.M.; Liu, S.T. Efficient oxidative coupling of 2,6-disubstituted phenol catalyzed by a dicopper (II) complex. Dalton Trans. 2012, 41, 1158-1164. [CrossRef] [PubMed]

27. Hideyuki, H.; Kyokaishi, Y.G.K. Radical-controlled oxidative polymerization of phenols. J. Synth. Org. Chem. Jpn. 2005, 63, 970-981. [CrossRef]

28. Uchidma, E.; Anko, M.; Ishide, H. The kinetics of the oxidative polymerization of 2.6-xylenol with a copper-amine complex. Makromol. Chem. 1972, 151, 221-234. [CrossRef]

29. Hay, A.S. Polymerization by oxidative coupling: Discovery and commercialization of PPO ${ }^{\circledR}$ and Noryl ${ }^{\circledR}$ resins. J. Polym. Sci. Part A Polym. Chem. 1998, 36, 505-517. [CrossRef]

30. Liu, Q.; Shentu, B.; Gu, C.; Weng, Z. The initial oxidative polymerization kinetics of 2,6-dimethylphenol with a Cu-EDTA complex in water. Eur. Polym. J. 2009, 45, 1080-1085. [CrossRef]

31. Kobayashi, S.; Higashimura, H. Oxidative polymerization of phenols revisited. Prog. Polym. Sci. 2003, 28, 1015-1048. [CrossRef]

(C) 2018 by the authors. Licensee MDPI, Basel, Switzerland. This article is an open access article distributed under the terms and conditions of the Creative Commons Attribution (CC BY) license (http://creativecommons.org/licenses/by/4.0/). 